\title{
Experimento para modelar a acomodação do olho humano
}

Experiment to model the accommodation of the human eye

\author{
Gustavo Trierveiler ${ }^{1}$, Julio Flemming*2@ \\ ${ }^{1}$ Colégio Estadual Alberto Gomes Veiga, Paranaguá, PR, Brasil \\ ${ }^{2}$ Universidade Estadual de Ponta Grossa, Departamento de Física, Ponta Grossa, PR, Brasil
}

Recebido em 17 de Abril, 2018. Revisado em 15 de Agosto, 2018. Aceito em 20 de Agosto, 2018.

\begin{abstract}
Construímos um sistema simples e barato que simula o processo de acomodação do olho humano. Utilizou-se uma lente bola flexível, feita de preservativo masculino de látex cheio de água, e dois lasers de diodo, que podem passar da condição de colimados a de divergentes, ajustando-se seus suportes rotatórios. O aparato serviu para aulas de óptica no ensino médio. Os educandos calcularam e mediram a distância focal da lente bola $\left(\mathrm{f}_{\text {medido }} / \mathrm{f}_{\text {calculado }}=\right.$ $0,94 \pm 0,06)$ e estudaram como a vista humana vai da condição de relaxada para a condição de focalizar objetos próximos, contraindo a lente (simulando o que faz o cristalino). Discute-se também outros detalhes da acomodação da vista humana.
\end{abstract}

Palavras-chave: visão humana, acomodação, cristalino, lentes flexíveis, lente bola.

We build a simple and inexpensive system that simulates the process of human accommodation. We used a ball lens, made of a male latex condom filled with water, and two diode lasers that can change from collimated to divergent beams, adjusting their rotatory supports. The device is suitable for secondary classes in optics. Students have calculated and measured the focal length of the ball lens $\left(\mathrm{f}_{\text {measured }} / \mathrm{f}_{\text {calculated }}=0.94 \pm 0.06\right)$ and have studied how human vision shifts from the relaxed condition to the condition of visualizing nearby objects, by squeezing the lens (simulating the crystalline work). Other details of human vision accommodation are also discussed.

Keywords: human vision, accommodation, crystalline. flexible lenses, ball lens.

\section{Introdução}

O estudo da Óptica é relevante tanto no ensino fundamental e médio como nos cursos universitários voltados para a área de exatas e mesmo de biológicas. Pode-se aumentar o interesse dos alunos sobre este tema analisando-se, por exemplo, o olho humano como um sistema óptico.

O olho humano é uma câmara de forma aproximadamente esférica, cercada por uma membrana opaca chamada de esclera. A estrutura se mantém devido a uma diferença positiva entre a pressão interna e a externa de cerca de $25 \mathrm{mmHg}$ (denominada de pressão manométrica do olho) [1]. A luz penetra internamente neste globo opaco por uma abertura frontal (a pupila) e, ao longo de seu trajeto, ela sofrerá várias refrações. A primeira e mais significativa refração ocorre na interface ar-córnea. Vide figura 1. Esta tem um raio de curvatura de cerca de $7,8 \mathrm{~mm}$, uma espessura próxima de $0,5 \mathrm{~mm}$ e um índice de refração de 1,376 . A seguir, o raio luminoso atravessa uma bolsa de espessura axial de cerca de 1,3mm contendo um líquido chamado de humor aquoso, com índice de refração de 1,336. Logo depois temos o cristalino, uma lente biconvexa de espessura próxima de $3,6 \mathrm{~mm}$ e raios de curvatura frontal e traseiro de $10 \mathrm{~mm}$ e $6 \mathrm{~mm}$, respectivamente. O índice de refração é crescente desde cerca de 1,37 até 1,42 , no sentido das bordas para o centro

*Endereço de correspondência: flemming@uepg.br.

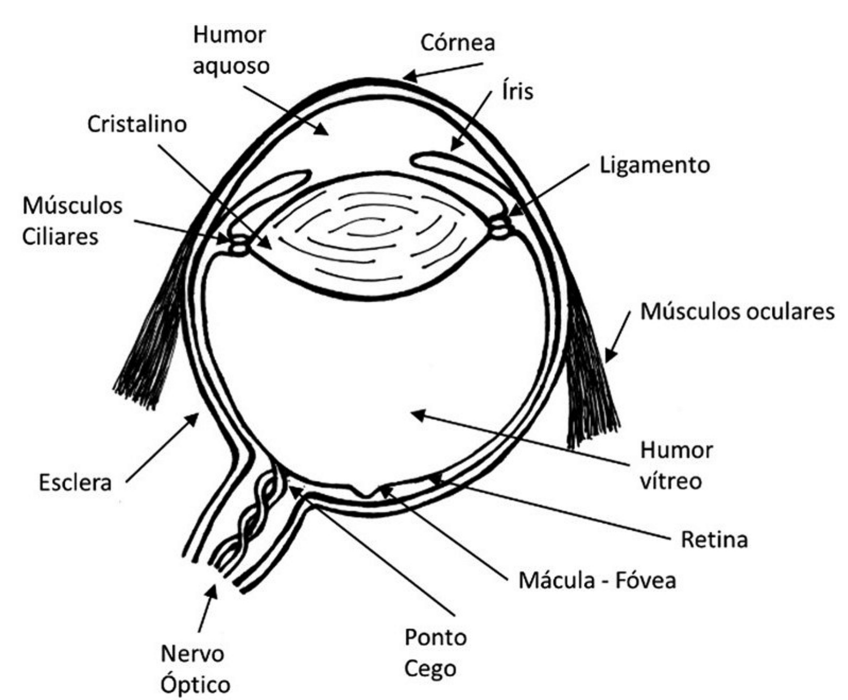

Figura 1: Principais partes do olho humano.

do cristalino. Em seguida, a luz encontra outra câmara contendo um líquido de índice de refração também próximo de 1,336, denominado humor vítreo. Finalmente, chegaremos à retina, que é um mosaico de fotorreceptores que transformarão a energia luminosa em sinais elétricos que serão enviados ao cérebro pelo sistema nervoso. 


\section{Considerações sobre a acomodação da vista humana}

A equação básica das lentes delgadas é a chamada equação de Gauss [2]:

$$
\frac{1}{f}=\frac{1}{o}+\frac{1}{i}
$$

onde $f$ é a distância focal da lente, o é a distância do objeto até a lente e $i$ é a distância da imagem até a lente. Esta equação só é válida para imagens nítidas, ou seja, "focalizadas". Uma imagem de um objeto qualquer que esteja borrada ou "desfocalizada", embora possa até nos dar uma ideia sobre como é o correspondente objeto, não obedece a esta equação e não será denominada, neste sentido estrito, de imagem. Percebemos que, um objeto (suposto plano e perpendicular ao eixo óptico) que mude sua posição ocom relação à lente terá sua correspondente imagem $i$ em outra posição, para uma lente que seja não flexível e que permaneça fixa. No entanto, frequentemente é desejável que a imagem (suposta real) permaneça imóvel, pois lá se encontra um anteparo, um filme sensível à luz ou fotodetectores. Para isto faz-se necessário, cada vez que a posição do objeto seja alterada, que haja um correspondente deslocamento da lente ou a mudança de sua distância focal $f$.No primeiro caso, alteraremos $i$ para satisfazer novamente a equação, com a imagem imóvel (apesar da imagem permanecer imóvel sobre o anteparo, $i$ é alterado pois ocorre o deslocamento da lente, que é a origem das coordenadas usadas na equação). Este processo é utilizado, por exemplo, nas câmaras fotográficas e filmadoras e, entre os seres vivos, em certos peixes [3]. No segundo caso, se a lente for flexível, tenho a possibilidade de alterar a distância focal $f$ dela. É o que acontece, por exemplo, em todas as aves e mamíferos como nós. O nosso olho possui uma lente biconvexa flexível chamada cristalino. Aumentando sua curvatura é possível diminuir sua distância focal $f$ (ou, equivalentemente, aumentar sua vergência $1 / f$ ) e focalizar objetos próximos. Este processo de mudança de forma do cristalino é chamado de acomodação. Quando a distância focal $f$ é medida em metros, a vergência é medida em dioptrias.

O poder de acomodação do olho é limitado. Uma pessoa com 30 anos consegue focalizar objetos distantes dela desde $12,5 \mathrm{~cm}$ até o infinito. Isto corresponde a uma amplitude de acomodação de 8 dioptrias [4]. A velocidade com que o olho consegue se acomodar é da ordem de 7 dioptrias por segundo [5], que resulta num tempo típico de 0,3 segundos, para focalizarmos uma paisagem distante quando tiramos a vista de uma tela de computador a $50 \mathrm{~cm}$. Isto é apenas um valor de referência, já que depende de vários fatores, como idade do indivíduo, se a acomodação é de perto para longe ou o contrário, visão bi ou monocular, se levamos em consideração o tempo de latência, etc. [6,7]. Interessante mencionar que algumas corujas têm uma velocidade de acomodação dez vezes maior que a humana [8].

Quando observamos objetos próximos, conjuntamente com a acomodação do cristalino ocorre a contração da pupila $[9,10]$. Isto serve para aumentar a chamada profundidade de campo, que evita que sejam necessários vários ajustes da distância focal conforme lemos, por exemplo. De fato, a profundidade de campo de uma lente pode ser definida como o quanto um objeto, do qual se tem uma imagem ideal (obedecendo precisamente à equação de Gauss), poderia variar sua posição e ainda assim fornecer uma imagem relativamente nítida, dentro de um limite arbitrado [1, p 332]. Esta profundidade de campo varia diretamente com o quadrado da distância do objeto $o$ e inversamente com o diâmetro da pupila [11]. Daí, como mencionado, a necessidade da contração desta para termos focalização estável de objetos próximos.

\section{Classificação do cristalino como lente}

Classifica-se as lentes como esféricas (versus asféricas), delgadas (versus espessas), singletos (versus multipletos) e trabalhando na aproximação paraxial (versus nãoparaxial), entre outras possíveis categorizações $[1$, p. 10 , 1, p. 402, 12].

As esféricas são aquelas em que as duas superfícies refratoras são calotas esféricas (podendo uma delas ser plana). Nas delgadas a separação entre estas superfícies é desprezível frente a seus raios de curvatura. Uma lente singleto é feita de um único material homogêneo, sem variação do índice de refração entre as superfícies refratoras. $\mathrm{Na}$ aproximação paraxial a luz incidente é praticamente paralela e muito próxima ao eixo óptico da lente. Isto permite simplificar as equações, pois senos e tangentes de ângulos relevantes podem ser aproximados pelos próprios ângulos. O cristalino humano é uma lente asférica [13] espessa e multipleto, pois temos uma diminuição progressiva do índice de refração em direção às bordas do cristalino.

Há infinitos tipos de superfícies tridimensionais asféricas (não esféricas). O tipo de asfericidade do cristalino varia se consideramos sua superfície anterior ou posterior, se ele está relaxado ou contraído e com a idade da pessoa [14]. Por exemplo, para a face anterior do cristalino, na sua acomodação máxima e para indivíduos na faixa de 30 anos a asfericidade é do tipo hiperbólica. Conforme variamos estes parâmetros, a asfericidade desta lente flexível pode assumir a forma de quádricas advindas da revolução de elipses, parábolas ou hipérboles.

Com relação ao cristalino ser uma lente espessa, observe que pode - se continuar usando a equação de Gauss para uma lente deste tipo, imersa no ar, desde que a origem das coordenadas seja agora os chamados planos principais $[1$, p. 35,12 , p. 257,15$]$. Na visão humana a aproximação paraxial é, em geral, válida. Desvios da aproximação paraxial causam aberrações geométricas, que correspondem, geralmente, a termos uma imagem não pontual (borrada) para um objeto pontual [1, p. 387, 16]. Parte destas aberrações são corrigidas pela asfericidade do cristalino e à variabilidade de seu índice de refração, mencionadas acima. Assim, de um modo geral, as aberrações são desprezíveis e pode-se usar a aproxi- 
mação paraxial no estudo da visão humana $[1$, p. 10, 1, p.426].

Nosso simulador, apresentado a seguir, assume uma série de aproximações. O olho humano real é reduzido a uma só lente no simulador, que faz o papel da superfície ar-córnea e do cristalino, já que estes realizam a maior parte da refração da luz e pelo último ser a parte de foco ajustável do olho. O cristalino real, que é uma lente espessa, é particularizado por uma lente espessa tipo "lente bola" no simulador, devido à maior facilidade experimental de se construir uma lente flexível deste tipo. Lentes bolas são aquelas com o formato de uma esfera $[17,18]$.

No nosso experimento, toda a refração da luz ocorre nas interfaces de entrada e saída da lente bola flexível, que representaria o cristalino. No olho humano, ao contrário, cerca de $70 \%$ da convergência está associada à interface de entrada ar-córnea (43 dioptrias). O cristalino relaxado adiciona 21 dioptrias $[13,18]$.

\section{Aparato experimental e sua utilização}

O aparato experimental utilizado faz parte de uma sequência didática formada por cinco momentos: estudo do raio de luz, construção de câmara escuras, estudo de lentes esféricas, estudo do olho (pesquisa e dissecação do olho de boi) e a construção do simulador didático do olho humano [19].

A elaboração da sequência didática foi baseada conforme a teoria de aprendizagem significativa de David Ausubel [20], com a elaboração de organizadores prévios, para só depois estudar o processo de acomodação do olho humano com o simulador didático.

O material é constituído por planos de aulas para o professor, material teórico para professores e alunos, atividades com questões e procedimentos experimentais que levam o aluno a investigar o olho humano, em especial, o comportamento da luz.

O aparato experimental, o simulador didático, emula a focalização de imagem dentro do globo ocular e o processo de acomodação do olho humano. Ele é formado por: uma lente flexível feita de preservativo masculino de látex preenchido com água; suportes para lente; fita métrica que representa a projeção do eixo óptico principal da lente flexível; modelo globo ocular com fundo olho humano (onde se encontra a retina) feito com uma semiesfera de isopor e dois lasers de diodo, de comprimento de onda entre 630 a $650 \mathrm{~nm}$, que representam os raios de luz incidentes sobre nossa lente. Para haver liberdade de alinhamento, eles ficam sustentados no topo de embalagens de desodorantes roll-on [19,21], que são preenchidas parcialmente com cimento, para maior estabilidade. Como também mostrado na figura $2 \mathrm{~A}$, a alimentação dos lasers é feita por pilhas comuns $(1,5 \mathrm{~V})$ em série, acondicionadas em um cano de PVC.

A escolha dos materiais tinha por pré-requisito a facilidade de aquisição e o baixo custo. O preservativo masculino, por exemplo, é fácil de ser adquirido em qual-

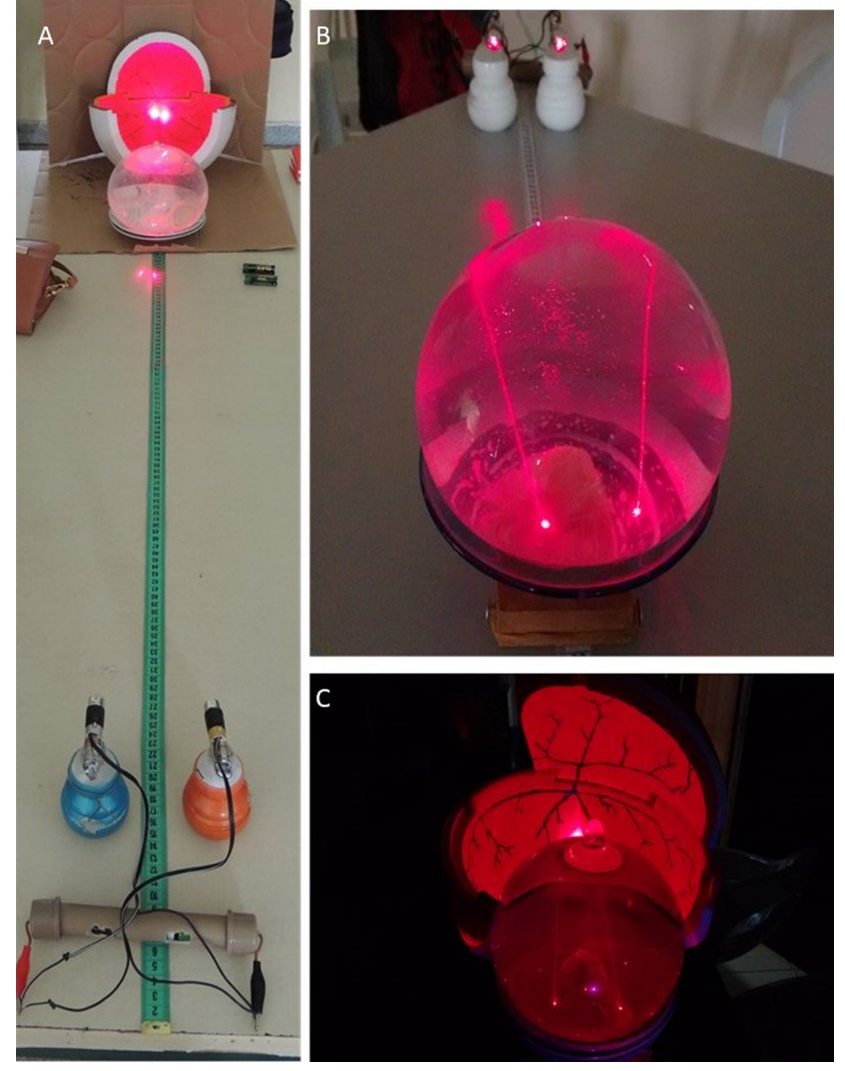

Figura 2: (A) Simulador didático montado pelos educandos. (B) Convergência dos raios de luz incidentes na lente bola. (C) Focalização dos raios de luz na "retina", no fundo do olho de isopor.

quer farmácia ou posto de saúde. Verificou-se que bexigas de festa não apresentam a mesma transparência, flexibilidade e resistência do preservativo.

O aparato experimental foi produzido para ser aplicado aos alunos da educação básica. No caso, em uma turma 42 alunos do curso de Técnico em Portos do Colégio Estadual Alberto Gomes Veiga, em Paranaguá, PR. A turma foi dividida em 12 equipes e cada uma montou o aparato experimental como mostrado na figura $2(\mathrm{~A})$. Os próprios educandos manusearam o equipamento e obtiveram as medidas necessárias, orientados por um roteiro, que continha a ordem dos procedimentos necessários para a execução da atividade. Além disso, cada equipe respondeu questões que incentivavam a observação e investigação, o que corrobora com o objetivo do produto educacional, que o aluno entenda como o olho humano focaliza a imagem de objeto em diferentes distâncias, através do processo de acomodação [19].

Antes da aplicação da atividade foram realizadas aulas [19] sobre a formação de imagens em câmaras escuras, estudo do olho e da equação de Gauss para lentes.

Em seguida as equipes simularam duas situações. A primeira com o feixe de luz paralelo a fita métrica, ou seja, paralelo à projeção do eixo óptico principal (fig.2(B)). Para isto, os alunos localizaram o eixo principal da lente flexível, posicionando o laser paralelamente a fita métrica 
e posicionando a lente, de tal forma, que o feixe de luz não sofra desvios, como mostrado na figura 3. Esta situação corresponde a um objeto localizado bastante longe do olho (no "infinito"). Há formação da imagem na retina com o cristalino relaxado.

A segunda situação corresponde a um objeto mais próximo do olho. Os raios de luz provenientes deste objeto são agora levemente divergentes e assim, correspondentemente, são posicionados os lasers. No olho humano, os chamados músculos ciliares devem exercer força para contrair o cristalino na proporção necessária para novamente termos a imagem na retina. No nosso experimento deve-se contrair adequadamente a lente flexível para simular o mesmo efeito. No primeiro caso foram obtidas as distâncias focais experimentais da lente flexível e o seu respectivo diâmetro e, na segunda situação, o educando deformava a lente flexível (comprimia), reduzindo sua distância focal original e reproduzindo o processo de acomodação do olho humano. Modelamos como ocorre a formação da imagem para raios luminosos que nem sempre são colimados, quando incidem nos nossos olhos.
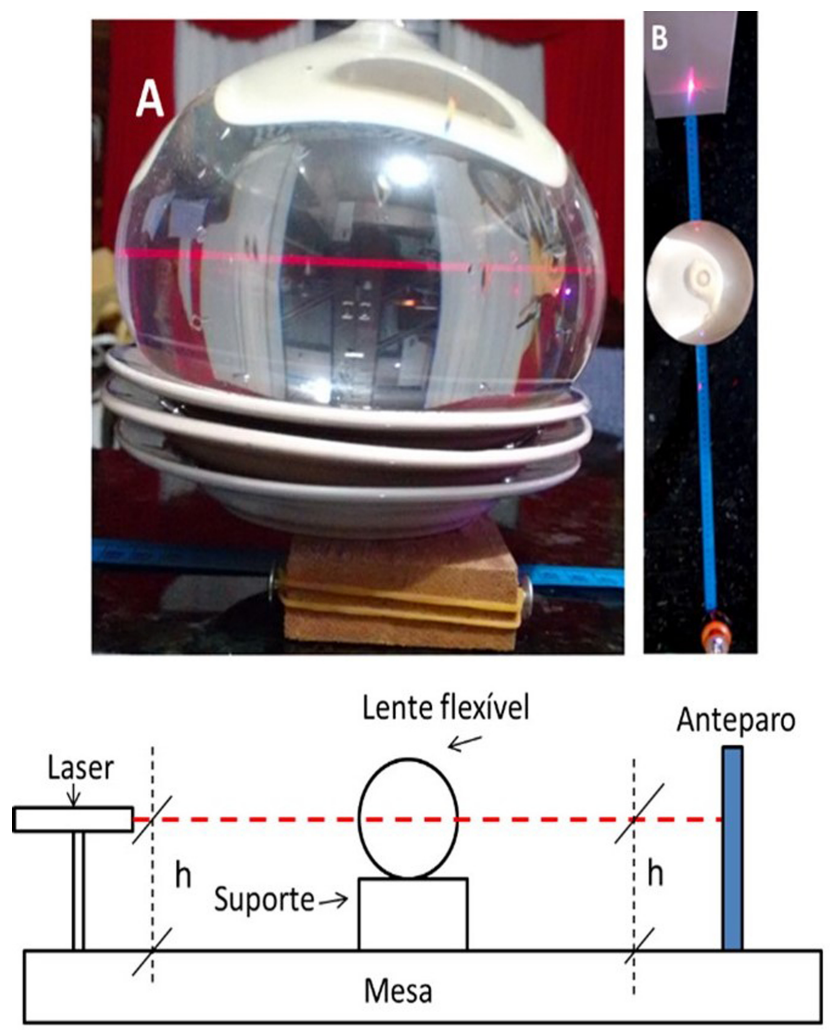

(C)

Figura 3: (A) O feixe de luz passando pelo eixo óptico da lente. Para melhor alinhamento usamos calços de madeira e pratos para apoiar a lente flexível. (B) O feixe de luz alinhado com a fita métrica e paralelo ao eixo principal, sem sofrer desvios. (C) Esquema explicativo do alinhamento do laser. Para facilitar o trabalho, usamos um anteparo e réguas para aferir as alturas "h". Se forem iguais significa que o feixe e luz passa pelo eixo principal da lente flexível.
Embora alguns trabalhos [22] mostrem a focalização de feixes divergentes, a maioria das figuras nos livros didáticos exibem somente feixes incidentes paralelos.

Nosso experimento permite que os alunos percebam na prática que, mesmo com feixes divergentes (por exemplo, quando lemos uma tela de celular), a imagem ainda pode ser formada na retina, devido à intervenção de uma lente flexível. Além disso, temos a possibilidade de simular os defeitos mais comuns da visão, como a miopia e a hipermetropia [1], que são advindas de uma distância não ideal da retina (desenhada no globo ocular de isopor, no nosso caso), em relação à lente bola relaxada.

Medindo-se a posição de convergência de raios de luz, que incidam paralelamente, obtêm-se a distância focal experimental ( $f_{\text {medido }}$ ) e a equação da lente bola permite o cálculo teórico desta $\left(\mathrm{f}_{\text {calculado }}\right)$. Nesta equação [22,23], representada abaixo, $d$ é o diâmetro da lente e $n$ o índice de refração do material com que ela é feita:

$$
f_{\text {teórico }}=\frac{n . d}{4(n-1)}
$$

No nosso caso, o material considerado é água $(n=1,33)$, pois não levamos em conta o índice de refração da borracha látex ou do óleo lubrificante que envolve o preservativo.

Como já mencionado, pode-se utilizar a equação de Gauss para uma lente espessa (como a lente bola), desde que a origem esteja nos denominados planos principais. $\mathrm{Na}$ lente bola os dois planos principais se fundem em um só: aquele que contém o centro da esfera e é perpendicular ao eixo óptico [1, p 35]. Na figura 4, está representado pelo pontilhado vertical que passa pelo ponto $\mathrm{O}$.

Cada equipe recebeu um roteiro que continha perguntas de cunho qualitativo e quantitativo. Eles mediram encheram os preservativos com valores aproximados de $15 \mathrm{~cm}$ e foram orientados a utilizarem como referência sua palma da mão.

Foram avaliados os erros experimentais calculando $\mathrm{f}_{\text {medido }} / \mathrm{f}_{\text {calculado }}$ e comparando quanto esta razão se afasta de um. Faz-se esta razão para cada equipe e, depois, tirase a média e o desvio padrão para todas as equipes. $\mathrm{O}$

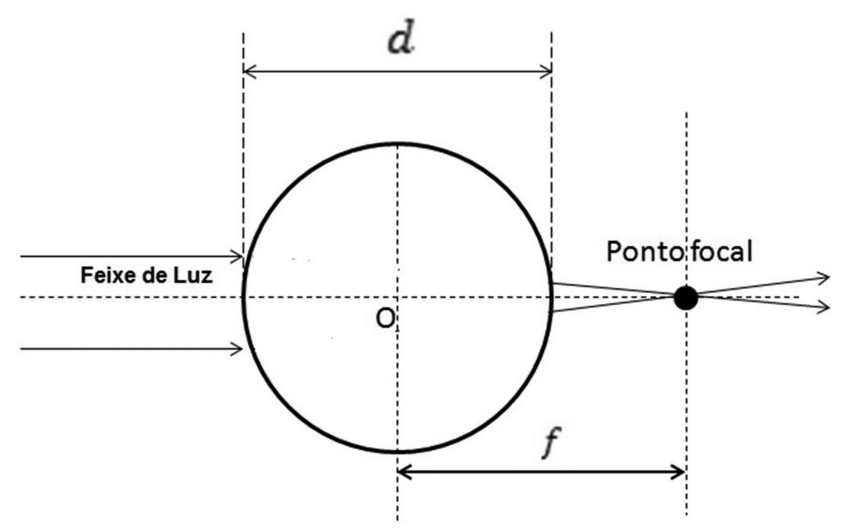

Figura 4: Representação da lente bola, com um feixe de luz colimado incidindo sobre ela. 
desvio padrão mostrará os erros aleatórios e a diferença (para mais ou para menos) com relação à unidade mostrará os erros sistemáticos. O resultado obtido com 12 equipes diferentes foi

$$
\frac{f_{\text {medido }}}{f_{\text {calculado }}}=0,94 \pm 0,06
$$

Isto mostra que o foco medido e o calculado são iguais, dentro da faixa de erro. Mas, de uma forma sistemática, a medição dá valores $6 \%$ abaixo do esperado teoricamente. $\mathrm{O}$ motivo pode ser o abaulamento da lente bola. Sendo feita com látex, ela se deforma sob a ação da gravidade, tornando-se aproximadamente um esferoide oblato. Esta é a superfície criada por uma elipse que revoluciona em torno de seu eixo menor. Desta forma a distância máxima na horizontal é um pouco maior que a correspondente na vertical, pois correspondem ao eixo maior e menor da elipse, respectivamente. Como os alunos mediram na horizontal, o diâmetro medido por eles é maior que o diâmetro médio de uma lente bola perfeita que aproxime nossa lente bola abaulada. Como a distância focal da lente bola é diretamente proporcional ao diâmetro (vide eq. (1)) ao se colocar este valor maior, a distância focal teórica ficaria incorretamente aumentada.

Durante a redação deste manuscrito, tomamos conhecimento de dois trabalhos [24,25] que guardam várias semelhanças com o nosso.

\section{Conclusão}

Este experimento simula o processo dinâmico de acomodação e relaxamento que ocorre, respectivamente, com raios divergentes ou colimados incidentes sobre a vista humana. É possível realizar-se certas medidas quantitativas e a utilização de uma semiesfera de isopor móvel onde se desenha a retina, além do apelo estético, permite mostrar os defeitos de miopia e hipermetropia. A construção e manuseio de um sistema simples que representa o olho humano, desenvolve as competências e habilidades dos educandos em pesquisar e construir aparatos experimentais e vão além da simples curiosidade, mas que são usados para obter e quantificar dados. Isto torna a aprendizagem significativa e incentiva o estudo da óptica.

\section{Agradecimentos}

A CAPES, aos alunos, professores e funcionários do Colégio Alberto Gomes Veiga da cidade de Paranaguá - PR, em especial à professora Maria Aparecida. Ao professor da UEPG Antônio Sérgio Magalhães de Castro.

\section{Referências}

[1] R.S. Longhurst, Geometrical and Physical Optics (Longman Group United Kingdom, Londres, 1974), $3^{\text {a }}$ ed.

[2] H.M. Nussenzveig, Curso de Física Básica (Ed. Edgard Blücher, São Paulo, 1998), 1 ${ }^{\mathrm{a}}$ ed., v. 4, p. 30.
[3] I.R. Schwab e N. Hart, British Journal of Ophthalmology 90, 406 (2006).

[4] W.N. Charman, Clinical and Experimental Optometry 91, 207 (2008).

[5] E. Chirre, P. Prieto e P. Artal, Biomedical Optics Express 6, 4200 (2015).

[6] G. Heron, W.N. Charman e C. Schaeffel, Vision Research 41, 507 (2001).

[7] F. Schaeffel, H. Wihelm e E. Zrenner, Journal of Physiology 461, 301 (1993).

[8] C.J. Murphy e H.C Howland, Journal of Comparative Physiology 151, 277 (1983).

[9] ENCYCLOPAEDIA Britannica (Encyclopædia Britannica, Londres, 1953), v. 23, p. 214.

[10] S. Kasthurirangan e A. Glasser, Ophthalmic and Physiological Optics 25, 328 (2005).

[11] L. Yung-Kuo, Problems and Solutions on Optics (World Scientific Publishing, Singapura, 1991) p.13.

[12] D. Gonçalves, Fúsica do Científico ao Vestibular (Ed. Ao livro Técnico, Rio de Janeiro, 1974), v. 4.

[13] R. Navarro, Journal of Optometry 2, 1 (2009).

[14] J. Birkenfeld, Optical and Structural Properties of the Crystalline Lens: Accommodation and Aging. Tese de Doutorado, Universidad Complutense de Madrid, Madrid (2014).

[15] M. Katz, Introduction to Geometrical Optics (World Scientific Publishing, Singapura, 2002).

[16] A.N. Matveev, Optics (Mirs Publishers, Moscow, 1988), p. 173.

[17] D. Soga, L.R.D. Paiva e M. Muramatsu, Revista Brasileira de Ensino de Física 39, e3506 (2017).

[18] http://hyperphysics.phy-astr.gsu.edu/hbase/ vision/eyescal.html\#c2, acessado em 26 04/2018.

[19] G.A. Trierveiler, Simulador Didático de Acomodação do Olho Humano. Tese de Mestrado, Universidade Estadual de Ponta Grossa, Ponta Grossa (2016).

[20] M.A. Moreira, Uma abordagem cognitivista ao ensino da física (Editora da UFRGS, Porto Alegre, 1983), $1^{\mathrm{a}} \mathrm{ed}$.

[21] E.G. Alves e A.F. da Silva, Física na Escola 9, 1 (2008).

[22] A.F. Guedes, L.F.M. Braun e M.R. Rizzatti, Revista Brasileira de Ensino de Física 19, 137 (2000).

[23] Understanding Ball Lenses, www.edmundoptics com/resources/application-notes/optics/ understanding-ball-lenses/, acessado em $26 / 04 / 2018$.

[24] V. Oommen e P. Kanthakumar, Adv. Physiology Education 38, 183 (2014).

[25] R.G. Antoniazi e M.M.G. Carvalho, Construção de sistema ótico a partir de lentes de água, disponível em https://www.ifi.unicamp.br/ lunazzi/ F530_F590_F690_F809_F895/F809/F809_sem2_2003/ 009849Rubens-Mauro_F809_RF.pdf , acessado em $14 / 08 / 2018$. 